\title{
Neutrinoless double-beta decay and seesaw mechanism
}

\author{
Samoil M. Bilenky, ${ }^{1,2}$ Amand Faessler, ${ }^{3}$ Walter Potzel, ${ }^{1}$ and Fedor Šimkovic ${ }^{2,4}$ \\ ${ }^{1}$ Physik-Department E15, Technische Universität München, D-85748 Garching, Germany \\ ${ }^{2}$ Laboratory of Theoretical Physics, JINR, 141980 Dubna, Moscow region, Russia \\ ${ }^{3}$ Institute of Theoretical Physics, University of Tuebingen, 72076 Tuebingen, Germany \\ ${ }^{4}$ Department of Nuclear Physics and Biophysics, Comenius University, \\ Mlynská dolina F1, SK-842 15 Bratislava, Slovakia
}

(Dated: May 27, 2018)

\begin{abstract}
From the standard seesaw mechanism of neutrino mass generation, which is based on the assumption that the lepton number is violated at a large $\left(\sim 10^{15} \mathrm{GeV}\right)$ scale, follows that the neutrinoless double-beta decay $(0 \nu \beta \beta$-decay) is ruled by the Majorana neutrino mass mechanism. Within this notion, for the inverted neutrino-mass hierarchy we derive allowed ranges of half-lives of the $0 \nu \beta \beta$-decay for nuclei of experimental interest with different sets of nuclear matrix elements. The present-day results of the calculation of the $0 \nu \beta \beta$-decay nuclear matrix elements are briefly discussed. We argue that if $0 \nu \beta \beta$-decay will be observed in future experiments sensitive to the effective Majorana mass in the inverted mass hierarchy region, a comparison of the derived ranges with measured half-lives will allow us to probe the standard seesaw mechanism assuming that future cosmological data will establish the sum of neutrino masses to be about $0.2 \mathrm{eV}$.
\end{abstract}

PACS numbers: 98.80.Es,23.40.Bw; 23.40.Hc

\section{INTRODUCTION}

The observation of neutrino oscillations in atmospheric [1], solar [2], reactor [3] and accelerator [4, 5] neutrino experiments is the most important recent discovery in particle physics. It is very unlikely that neutrino masses, many orders of magnitude smaller than the masses of quarks and leptons, are generated by the standard Higgs mechanism. Small neutrino masses and neutrino mixing are commonly considered as a signature of physics beyond the Standard Model (SM). Several beyond the SM mechanisms of neutrino mass generation were proposed. The most viable and plausible mechanism is the famous seesaw mechanism which is based on the assumption that the total lepton number $L$ is violated at a scale much larger than the electroweak scale $v=\left(\sqrt{2} G_{F}\right)^{-1 / 2} \simeq 246$ $\mathrm{GeV}$.

If the total lepton number is violated, neutrinos $\nu_{i}$ with definite masses are Majorana particles. After the discovery of neutrino oscillations the problem of the nature of neutrinos with definite masses (Majorana or Dirac?) became the most pressing issue.

Information about the nature of neutrinos with definite masses can not be obtained via the investigation of neutrino oscillations [6]. In order to obtain such an information it is necessary to study processes in which the total lepton number $L$ is violated. The investigation of neutrinoless double-beta decay ( $0 \nu \beta \beta$-decay) of eveneven nuclei

$$
(A, Z) \rightarrow(A, Z+2)+e^{-}+e^{-}
$$

is the most sensitive way to search for the effects of the lepton number violation.

The observation of the $0 \nu \beta \beta$-decay will prove that $\nu_{i}$ are Majorana particles. In this paper we show that an evidence of this process in future $0 \nu \beta \beta$-decay experiments sensitive to the effective Majorana mass in the inverted mass hierarchy region could allow us to obtain information about the validity of the original seesaw idea [7] of neutrino mass generation associated with a violation of the total lepton number at GUT scale assuming that a proper information about the lightest neutrino mass will be available from future cosmological data.

\section{SEESAW MECHANISM OF NEUTRINO MASS GENERATION}

The standard seesaw mechanism (type I seesaw) is based on the assumption that there exist heavy Majorana leptons $N_{i}$, singlets of the $S U_{L}(2) \times U(1)$ group, which have the following lepton number violating Yukawa interactions with lepton and Higgs doublets

$$
\mathcal{L}=-\sqrt{2} \sum_{i, l} Y_{l i} \bar{L}_{l L} N_{i R} \tilde{H}+\text { h.c.. }
$$

Here

$$
L_{l L}=\left(\begin{array}{c}
\nu_{l L} \\
l_{L}
\end{array}\right), \quad H=\left(\begin{array}{c}
H^{(+)} \\
H^{(0)}
\end{array}\right)
$$

are lepton and Higgs doublets, $\tilde{H}=i \tau_{2} H^{*}, Y_{i l}$ are dimensionless constants and

$$
N_{i}=N_{i}^{c}=C \bar{N}_{i}^{T}
$$

is the field of heavy Majorana leptons with mass $M_{i}$ which is much larger than $v$.

At electroweak energies for the processes with virtual $N_{i}$ the interactions (11) generate the effective Lagrangian

$$
\mathcal{L}_{\text {eff }}=-\frac{1}{\Lambda} \sum_{l^{\prime}, l, i} \bar{L}_{l^{\prime} L} \tilde{H} \sum_{i}\left(Y_{l^{\prime} i} \frac{\Lambda}{M_{i}} Y_{l i}\right) C \tilde{H}^{T}\left(\bar{L}_{l L}\right)^{T}+\text { h.c. }
$$


which does not conserve the total lepton number $L$ and is the only effective Lagrangian of the dimension five [8]. In (3), the parameter $\Lambda$ has the dimension of mass and characterizes the scale of new physics beyond the SM.

After spontaneous violation of the electroweak symmetry the Lagrangian (3) generates the left-handed $\mathrm{Ma}$ jorana mass term

$$
\mathcal{L}^{\mathrm{M}}=-\frac{1}{2} \sum_{l^{\prime}, l} \bar{\nu}_{l^{\prime} L} M_{l^{\prime} l}^{L}\left(\nu_{l L}\right)^{c}+\text { h.c. }=-\frac{1}{2} \sum_{i} m_{i} \bar{\nu}_{i} \nu_{i}
$$

where

$$
M^{L}=Y \frac{v^{2}}{M} Y^{T}=U m U^{T},
$$

$\nu_{i}^{c}=\nu_{i}$ is the field of the Majorana neutrino with the mass $m_{i}$ and the flavor field $\nu_{l L}$ is given by the standard mixing relation

$$
\nu_{l L}=\sum_{i} U_{l i} \nu_{i L}
$$

Here, $U_{l i}$ are the elements of the Pontecorvo-MakiNakagawa-Sakata neutrino mixing matrix [9, 10]. The size of neutrino masses is determined by the seesaw factor $\frac{v^{2}}{M_{i}}$. From the existing data we can estimate that $M_{i} \simeq\left(10^{14}-10^{15}\right) \mathrm{GeV}$.

Let us stress that from the point of view of the standard seesaw approach small Majorana neutrino masses are the only low energy signature of physics beyond the SM at a GUT scale where the total lepton number is violated 1 1

The effective Lagrangian $\mathcal{L}_{\text {eff }}$ and, consequently, the left-handed Majorana mass term (41) can be generated not only by the interaction (1) but also by an interaction of lepton pairs and a Higgs pair with a triplet heavy scalar boson $\Delta$ (type II seesaw) and by an interaction of lepton-Higgs pairs with heavy Majorana triplet fermion $\Sigma$ (type III seesaw) (see [12]).

From the previous discussion we can conclude that if the total lepton number $L$ is violated at a GUT scale due to the existence of a heavy singlet (or triplet) Majorana fermion or a heavy triplet scalar boson interacting with standard lepton and Higgs doublets then

- neutrinos have small, seesaw suppressed masses (in accordance with the existing experimental data),

- neutrinos with definite masses are Majorana particles and the only mechanism of the $0 \nu \beta \beta$-decay is the exchange of virtual Majorana neutrinos.

In this paper we will explore these just mentioned general consequence of the standard seesaw mechanism.

\footnotetext{
1 In the early Universe at very high temperatures heavy Majorana leptons $N_{i}$ can be produced. Their $C P$-violating decays could lead to the baryon asymmetry of the Universe (see [11] and references therein).
}

\section{III. $0 \nu \beta \beta$-DECAY: NUCLEAR MATRIX ELEMENTS}

Neutrinoless double $\beta$-decay of even-even nuclei is a process of second order in the Fermi constant $G_{F}$ with the exchange of virtual Majorana neutrinos between $n-$ $p-e^{-}$vertexes. The mixed neutrino propagator has the form

$$
\begin{aligned}
& \sum_{i} U_{e i}^{2}\left(\frac{1-\gamma_{5}}{2}\right) \frac{\gamma \cdot p+m_{i}}{p^{2}-m_{i}^{2}}\left(\frac{1-\gamma_{5}}{2}\right) C \\
& \simeq m_{\beta \beta} \frac{1}{p^{2}}\left(\frac{1-\gamma_{5}}{2}\right) C,
\end{aligned}
$$

where

$$
m_{\beta \beta}=\sum_{i} U_{e i}^{2} m_{i}
$$

is the effective Majorana mass.

Let us stress that

- due to the $V-A$ structure of the weak charged current and neutrino mixing the matrix elements of the $0 \nu \beta \beta$-decay are proportional to $m_{\beta \beta}$.

- the average momentum of the virtual neutrinos is about $100 \mathrm{MeV}$. Thus, $p^{2} \gg m_{i}^{2}$ and the nuclear matrix elements do not depend on $m_{i}$. As a result, in the matrix elements of the $0 \nu \beta \beta$-decay, neutrino properties and nuclear properties are factorized.

The inverted half-life of the $0 \nu \beta \beta$-decay is given by the following general expression [13]

$$
\frac{1}{T_{1 / 2}^{0 \nu}(A, Z)}=\left|m_{\beta \beta}\right|^{2}|M(A, Z)|^{2} G^{0 \nu}\left(E_{0}, Z\right) .
$$

Here $M(A, Z)$ is the nuclear matrix element (NME) (matrix element between states of the initial and the final nuclei of the integrated product of two hadron charged currents and the neutrino propagator) and $G^{0 \nu}\left(E_{0}, Z\right)$ is a known phase-space factor ( $E_{0}$ is the energy release) 2

For our calculations we will need the values of the $0 \nu \beta \beta$-decay NMEs for different nuclei of experimental interest. We will briefly discuss here the present-day situation of the calculation of NMEs, compare existing methods, stress differences between them and present current values of NMEs.

The calculation of the NME is a complicated nuclear many-body problem. During many years two approaches were used: the Quasiparticle Random Phase Approximation (QRPA) 15-17] and the Interacting Shell Model (ISM) [18]. There are substantial differences between both approaches. The QRPA treats a large single particle

\footnotetext{
${ }^{2}$ For numerical values of $G^{0 \nu}\left(E_{0}, Z\right)$ see 14 .
} 
TABLE I. The NME of the $0 \nu \beta \beta$-decay calculated in the framework of different approaches: interacting shell model (ISM) [18], quasiparticle random phase approximation (QRPA) [15, 17], projected Hartree-Fock Bogoliubov approach (PHFB, PQQ2 parametrization) ) [20], energy density functional method (EDF) [22] and interacting boson model (IBM) 221]. The MillerSpencer Jastrow two-nucleon short-range correlations are taken into account. The EDF results are multiplied by 0.80 in order to account for the difference between UCOM and Jastrow [16]. $g_{A}=1.25$ and $R=1.2 A^{1 / 3}$ are assumed.

\begin{tabular}{lccccc}
\hline \hline Transition & \multicolumn{5}{c}{$M(A, Z)$} \\
\cline { 2 - 6 } & ISM [18] & QRPA [15, 17] & IBM-2 [21] PHFB [20] & EDF [22] \\
\hline${ }^{48} \mathrm{Ca} \rightarrow{ }^{48} \mathrm{Ti}$ & 0.61 & & & & 1.91 \\
${ }^{76} \mathrm{Ge} \rightarrow{ }^{72} \mathrm{Se}$ & 2.30 & 4.92 & 5.47 & & 3.70 \\
${ }^{82} \mathrm{Se} \rightarrow{ }^{82} \mathrm{Kr}$ & 2.18 & 4.39 & 4.41 & & 3.39 \\
${ }^{96} \mathrm{Zr} \rightarrow{ }^{96} \mathrm{Mo}$ & & 1.22 & & 2.78 & 4.54 \\
${ }^{100} \mathrm{Mo} \rightarrow{ }^{100} \mathrm{Ru}$ & & 3.64 & 3.73 & 6.55 & 4.08 \\
${ }^{116} \mathrm{Cd} \rightarrow{ }^{116} \mathrm{Sn}$ & & 2.99 & & & 3.80 \\
${ }^{124} \mathrm{Sn} \rightarrow{ }^{124} \mathrm{Te}$ & 2.10 & & & & 3.87 \\
${ }^{128} \mathrm{Te} \rightarrow{ }^{128} \mathrm{Xe}$ & 2.34 & 3.97 & 4.52 & 3.89 & 3.30 \\
${ }^{130} \mathrm{Te} \rightarrow{ }^{130} \mathrm{Xe}$ & 2.12 & 3.56 & 4.06 & 4.36 & 4.12 \\
${ }^{136} \mathrm{Xe} \rightarrow{ }^{136} \mathrm{Ba}$ & 1.76 & 2.30 & & & 3.38 \\
${ }^{150} \mathrm{Nd} \rightarrow{ }^{150} \mathrm{Sm}$ & & 3.16 & 2.32 & 3.16 & 1.37 \\
\hline \hline
\end{tabular}

model space, but truncates heavily the included configurations. The ISM, by contrast, treats a small fraction of this model space, but allows the nucleons to correlate in many different ways. We note that the latest QRPA results of the Jyväskylä-La Plata group [19] agree well with those of the Tuebingen-Bratislava-Caltech group [15, 16] by using the same way of adjusting the parameters of the nuclear Hamiltonian [15].

In the last few years several new approaches have been used for the calculation of the $0 \nu \beta \beta$-decay NMEs: the angular momentum Projected Hartree-Fock-Bogoliubov method (PHFB) 20], the Interacting Boson Model (IBM) 21], and the Energy Density Functional method (EDF) [22]. In the PHFB approach, the nucleon pairs different from $0^{+}$in the intrinsic coordinate system are strongly suppressed. In the framework of the ISM and the QRPA approaches it was shown, however, that other neutron pairs make significant contributions [23]. Let us notice also that in the IBM approach only transitions of $0^{+}$and $2^{+}$neutron pairs into proton pairs are taken into account. The EDF approach is an improvement with respect to the PHFB approach. Beyond-mean-field effects are included within the generating coordinate method with particle number and angular momentum projection for both initial and final ground states. But, the quality of the IBM and the EDF many-body wave functions have not been tested yet by the calculation of the $2 \nu \beta \beta$-decay half-lives.

In Table I, recent results of the different methods are summarized. The presented numbers have been obtained with the unquenched value of the axial coupling constant $\left(g_{A}=1.25\right)^{3}$, Miller-Spencer Jastrow short-range correlations 24] (the EDF values are multiplied by 0.80 in or-

3 A modern value of the axial-vector coupling constant is $g_{A}=$ 1.269. We note that in the referred calculations of the $0 \nu \beta \beta$ - der to account for the difference between the unitary correlation operator method (UCOM) and the Jastrow approach [16]), the same nucleon dipole form-factors, higher order corrections to the nucleon current and the nuclear radius $R=r_{0} A^{1 / 3}$, with $r_{0}=1.2 \mathrm{fm}$ (the QRPA values [15] for $r_{0}=1.1 \mathrm{fm}$ are rescaled with the factor 1.2/1.1). Thus, the discrepancies among the results of different approaches are solely related to the approximations on which a given nuclear many-body method is based.

From Table $\square$ we see that the smallest values of NMEs are obtained in the ISM approach. They are by about a factor of 2-3 smaller in comparison with results of other methods. The largest values of NME are obtained in the IBM $\left({ }^{76} \mathrm{Ge}\right.$ and $\left.{ }^{128} \mathrm{Te}\right)$, PHFB $\left({ }^{100} \mathrm{Mo},{ }^{130} \mathrm{Te}\right.$ and ${ }^{150} \mathrm{Nd}$ ), QRPA $\left({ }^{150} \mathrm{Nd}\right)$ and $\mathrm{EDF}\left({ }^{48} \mathrm{Ca},{ }^{96} \mathrm{Zr},{ }^{116} \mathrm{Cd}\right.$, ${ }^{124} \mathrm{Sn}$ and ${ }^{136} \mathrm{Xe}$ ) approaches. NMEs obtained by the QRPA and IBM methods are in a good agreement (with the exception of ${ }^{150} N d$ ). It is remarkable that for ${ }^{130} \mathrm{Te}$ the results of four different methods (QRPA, PHFB, IBM and EDF) are close to each other.

The differences among the listed methods of NME calculations for the $0 \nu \beta \beta$-decay are due to the following reasons:

(i) The mean field is used in different ways. As a result, single particle occupancies of individual orbits of various methods differ significantly from each other 25].

(ii) The residual interactions are of various origin and renormalized in different ways.

(iii) Various sizes of the model space are taken into account.

(iv) Different many-body approximations are used in the diagonalization of the nuclear Hamiltonian.

decay NMEs the previously accepted value $g_{A}=1.25$ was assumed. 


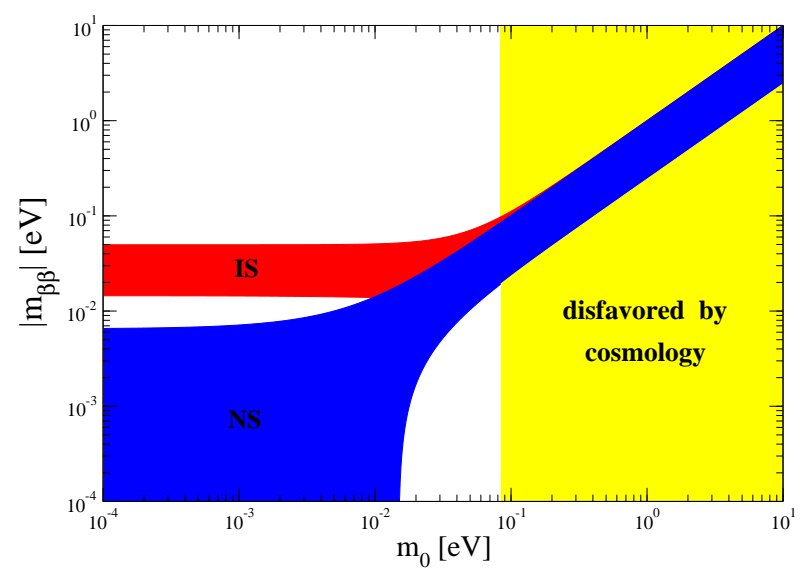

FIG. 1. (Color online) Effective Majorana neutrino mass $\left|m_{\beta \beta}\right|$ as function of the lightest neutrino mass $m_{0}$ for the cases of normal (NS, $m_{0}=m_{1}$ ) and inverted (IS, $m_{0}=m_{3}$ ) spectrum of neutrino masses. $\Delta m_{A}^{2}=(2.43 \pm 0.13) \times 10^{-3} \mathrm{eV}^{2}$ 5], $\Delta m_{S}^{2}=\left(7.65_{-0.20}^{+0.13}\right) \times 10^{-5} \mathrm{eV}^{2}$ 37], $\tan ^{2} \theta_{12}=0.452_{-0.033}^{+0.035}$ [3] and $0.03(0.04)<\sin ^{2} 2 \theta_{13}<0.28(0.34)$ 38] for NS (IS) are considered. The current limit of $\sum_{i=1}^{3} m_{i} \leq 0.28 \mathrm{eV}$ [39] for the sum of neutrino masses excludes values of $m_{0}$ larger 0.084 $\mathrm{eV}$.

Each of the applied methods has some advantages and disadvantages.

\section{POSSIBLE PROBE OF THE MAJORANA NEUTRINO MASS MECHANISM OF THE $0 \nu \beta \beta$-DECAY}

Many experiments on the search for $0 \nu \beta \beta$-beta decay of different nuclei were performed (see [26]). No indications in favor of $0 \nu \beta \beta$-decay were obtained in these experiments. There exist, however, a claim of the observation of the $0 \nu \beta \beta$-decay of ${ }^{76} \mathrm{Ge}$ made by some participants of the Heidelberg-Moscow collaboration [27]. Their estimated value of the effective Majorana mass (assuming a specific value for the NME) is $\left|m_{\beta \beta}\right| \simeq 0.4 \mathrm{eV}$. This result will be checked by an independent experiment relatively soon. In the new germanium experiment GERDA [28], the Heidelberg-Moscow sensitivity will be reached in about one year of measuring time.

From the most precise experiments on the search for $0 \nu \beta \beta$-decay the following bounds were inferred [29 31]:

$$
\begin{aligned}
\left|m_{\beta \beta}\right| & <(0.20-0.32) \mathrm{eV} \quad\left({ }^{76} \mathrm{Ge}\right) \\
& <(0.30-0.71) \mathrm{eV} \quad\left({ }^{130} \mathrm{Te}\right) \\
& <(0.50-0.96) \mathrm{eV} \quad\left({ }^{130} \mathrm{Mo}\right)
\end{aligned}
$$

These bounds we obtained using the $0 \nu \beta \beta$-decay NMEs of 32] calculated with Brueckner two-nucleon shortrange correlations.
In future experiments, CUORE 30], EXO 33, MAJORANA 34], SuperNEMO 35], SNO+ [36], Kamland-ZEN and others [26], a sensitivity

$$
\left|m_{\beta \beta}\right| \simeq \mathrm{a} \text { few } 10^{-2} \mathrm{eV}
$$

is planned to be reached.

The value of the effective Majorana mass strongly depends on the character of the neutrino mass spectrum. For the case of three neutrinos two types of mass spectra are allowed by the neutrino oscillation data:

1. Normal spectrum (NS)

$$
m_{1}<m_{2}<m_{3}, \quad \Delta m_{12}^{2} \ll \Delta m_{23}^{2},
$$

2. Inverted spectrum (IS)

$$
m_{3}<m_{1}<m_{2}, \quad \Delta m_{12}^{2} \ll\left|\Delta m_{13}^{2}\right| .
$$

In the case of NS, we have for the neutrino masses

$$
m_{2}=\sqrt{m_{0}^{2}+\Delta m_{S}^{2}}, \quad m_{3} \simeq \sqrt{m_{0}^{2}+\Delta m_{A}^{2}} .
$$

For IS we find

$$
m_{1}=\sqrt{m_{0}^{2}+\Delta m_{A}^{2}}, \quad m_{2} \simeq \sqrt{m_{0}^{2}+\Delta m_{A}^{2}} .
$$

Here $\Delta m_{12}^{2}=\Delta m_{S}^{2}$ and $\Delta m_{23}^{2}\left(\left|\Delta m_{13}^{2}\right|\right)=\Delta m_{A}^{2}$ are the solar and atmospheric neutrino mass-squared differences, respectively, and $m_{0}=m_{1}\left(m_{3}\right)$ is the lightest neutrino mass for NS(IS).

In Fig. 1 the effective Majorana mass $\left|m_{\beta \beta}\right|$ is plotted as a function of $m_{0}$ for the cases of the NS and the IS4. The lowest value for the sum of the neutrino masses, which can be reached in future cosmological measurements [39 41], is about (0.05-0.1) eV. The corresponding values of $m_{0}$ are in the region, where the IS and the NS predictions for $\left|m_{\beta \beta}\right|$ differ significantly from each other.

Future experiments on the search for the $0 \nu \beta \beta$-decay will probe the region of the inverted mass hierarchy 5

$$
m_{3} \ll m_{1}<m_{2} .
$$

In this case we have

$$
m_{1} \simeq m_{2} \simeq \sqrt{\Delta m_{A}^{2}}, \quad m_{3} \ll \sqrt{\Delta m_{A}^{2}} .
$$

Neglecting small contribution of the term $m_{3}\left|U_{e 3}\right|^{2}$, for the effective Majorana mass in the case of the inverted mass hierarchy (16) we obtain the following expression

$$
\left|m_{\beta \beta}\right| \simeq \sqrt{\Delta m_{A}^{2}} \cos ^{2} \theta_{13}\left(1-\sin ^{2} 2 \theta_{12} \sin ^{2} \alpha_{12}\right)^{\frac{1}{2}}
$$

\footnotetext{
4 Notice that for NS this figure differs significantly from analogous figures published in the literature. This is connected with the fact that we use new T2K data [38] for the values of the parameter $\sin ^{2} 2 \theta_{13}$.

${ }^{5}$ Let us note that the $0 \nu \beta \beta$-decay in the case of the inverted hierarchy was considered in detail in a recent paper [42].
} 
where $\alpha_{12}=\alpha_{2}-\alpha_{1}$ is the difference of the Majorana phases of the elements $U_{e 2}$ and $U_{e 1}$ with $U_{e i}=\left|U_{e 1}\right| e^{i \alpha_{i}}$ $(\mathrm{i}=1,2)$.

The phase difference $\alpha_{12}$ is the only unknown parameter in the expression for $\left|m_{\beta \beta}\right|$. From (18) we obtain the following inequality

$$
\sqrt{\Delta m_{A}^{2}} \cos ^{2} \theta_{13} \cos 2 \theta_{12} \leq\left|m_{\beta \beta}\right| \leq \sqrt{\Delta m_{A}^{2}} \cos ^{2} \theta_{13},
$$

where upper and lower bounds correspond to the case of the $C P$ invariance in the lepton sector (upper (lower) bound corresponds to the same (opposite) $C P$-parities of $\nu_{1}$ and $\left.\nu_{2}\right)$.

From (19) we find

$$
1.5 \cdot 10^{-2} \mathrm{eV} \leq\left|m_{\beta \beta}\right| \leq 5.0 \cdot 10^{-2} \mathrm{eV},
$$

where we used the MINOS value $\Delta m_{A}^{2}=(2.43 \pm 0.13) \times$ $10^{-3} \mathrm{eV}^{2}$ [5], the solar-KamLAND value $\tan ^{2} \theta_{12}=$ $0.452_{-0.033}^{+0.035}[3]$ and the recent $\mathrm{T} 2 \mathrm{~K}$ observation $\theta_{13}$ : $0.04<\sin ^{2} 2 \theta_{13}<0.34$ 38.

From (17) follows that in the case of inverted mass hierarchy we have for the sum of the neutrino masses

$$
\sum_{i=1}^{3} m_{i} \simeq 2 \sqrt{\Delta m_{A}^{2}} \simeq 10^{-1} \mathrm{eV} .
$$

As is well known, the quantity $\sum_{i} m_{i}$ can be inferred from the measurement of the distribution of galaxies and other cosmological observations. At present from cosmological data the bound $\sum_{i} m_{i} \lesssim 0.5 \mathrm{eV}$ was obtained [43], 39]. It is expected that in the future various cosmological observables will be sensitive to $\sum_{i} m_{i}$ in the range $\left(6 \cdot 10^{-3}-10^{-1}\right) \mathrm{eV}$ (see, for example, [41]). Thus, the inverted neutrino mass hierarchy (16) will be tested by future precision cosmology.

We will now discuss a possibility to check the Majorana mass mechanism for the case that the $0 \nu \beta \beta$-decay will be observed in future experiments sensitive to the region (20) of the inverted hierarchy.

From (9) and (19) we find the following inequalities for the half-life of $0 \nu \beta \beta$-decay

$$
T_{1 / 2}^{\min }(A, Z) \leq T_{1 / 2}^{0 \nu}(A, Z) \leq T_{1 / 2}^{\max }(A, Z)
$$

with

$$
\begin{aligned}
T_{1 / 2}^{\min }(A, Z) & =\frac{1}{\Delta m_{A}^{2}|M(A, Z)|^{2} G^{0 \nu}\left(E_{0}, Z\right)}, \\
T_{1 / 2}^{\max }(A, Z) & =\frac{1}{\Delta m_{A}^{2} \cos ^{2} 2 \theta_{12}|M(A, Z)|^{2} G^{0 \nu}\left(E_{0}, Z\right)} .
\end{aligned}
$$

In Fig 2 we present ranges of $0 \nu \beta \beta$-decay half-lives of different nuclei. If the measured half-life of the $0 \nu \beta \beta$-decay is in the range given by Eq. (22) this will be an evidence in favor of the Majorana neutrino mass mechanism (assuming inverted mass hierarchy).

Two remarks are in order:
1. It is seen from Fig 1 that the horizontal band determined by the inequality (20) is restricted by the condition $m_{0} \leq \sqrt{\Delta m_{A}^{2}} \simeq 5 \cdot 10^{-2} \mathrm{eV}$ (which corresponds to $\sum_{i} m_{i} \leq 1.9 \cdot 10^{-1} \mathrm{eV}$ ). If future cosmological data will establish this range for $m_{0}$ and the measured $0 \nu \beta \beta$-decay half-lives will be within the range given by inequality (22) this will be an evidence in favor of the Majorana mass mechanism. It is obvious that without knowledge of the value of the lightest neutrino mass it is impossible to determine in which region we are (IS or NS).

2. In addition to the Majorana mass mechanism, also other mechanisms of the $0 \nu \beta \beta$-decay, caused by a possible violation of the total lepton number $L$ at a scale which is much smaller than the standard seesaw GUT scale, were discussed in the literature. If $L$ is violated at $\sim \mathrm{TeV}$ scale a contribution of these additional mechanisms to the matrix element of the $0 \nu \beta \beta$-decay can be comparable with the Majorana mass contribution 6 . Thus, a significant violation of the inequalities (22) could happen. Examples of new mechanisms are the exchange of heavy Majorana neutralino or gluino in SUSY models with the violation of the R-parity (see recent papers [4548]), the exchange of right-handed Majorana neutrinos with mass at the electroweak scale (see 49]), the exchange of heavy Majorana right-handed neutrinos in $L-R$ models (see [44]), etc.

New mechanisms are characterized by parameters which are not connected with neutrino oscillation parameters. In addition, the NMEs of the Majorana mass mechanism and these possibly additional mechanisms are not connected and are different. Thus, it requires fine tuning for such mechanisms to contribute to the relatively narrow Majorana mass region (20) and mimic the effect of the Majorana phase difference. It is natural to expect that contributions to the matrix element of the $0 \nu \beta \beta$-decay of the Majorana mass mechanism and mechanisms connected with the violation of the lepton number at $\mathrm{TeV}$ scale could be quite different (see, for example, [49, [50]). Moreover, such mechanisms will be checked in LHC experiments by the search for effects of a violation of the total lepton number (like production of the same-sign lepton pairs in $p-p$ collisions, etc., see [50]).

${ }^{6}$ In fact, the matrix element of the $0 \nu \beta \beta$-decay in the case of the Majorana neutrino mass mechanism is proportional to $\left(\frac{G_{F}}{\sqrt{2}}\right)^{2} \frac{m_{\beta \beta}}{p^{2}}$, where $p \simeq 100 \mathrm{MeV}$ is the average momentum of the virtual neutrino. On the other side, the contribution to the matrix element of the $0 \nu \beta \beta$-decay by an exchange of a heavy Majorana lepton with a mass $M_{\chi} \sim \Lambda$ ( $\Lambda$ is the scale of new physics $)$ is proportional to $\left(\frac{G_{F}}{\sqrt{2}}\right)^{2}\left(\frac{M_{W}^{4}}{\Lambda^{5}}\right)\left(M_{W}\right.$ is the mass of the W-boson) [4]]. If we assume that $\left|m_{\beta \beta}\right| \simeq 10^{-2} \mathrm{eV}$ we conclude that both contributions are comparable, if $M_{\chi} \simeq 2 \mathrm{TeV}$. 
In Fig 2 we present ranges of $0 \nu \beta \beta$-decay half-lives of different nuclei in the case of inverted hierarchy of the neutrino masses. Three sets of NMEs are considered:

1) The ISM NMEs [18 calculated for $g_{A}=1.25$ (see second column of Table 【). In this case $M(A, Z)=M^{\min }(A, Z)=M^{\max }(A, Z)$.

2) The minimal $M^{\min }(A, Z)$ and maximal $M^{\max }(A, Z)$ NMEs of the QRPA, IBM and EDF approaches (see Table I).

3) The NMEs calculated in the framework of the renormalized QRPA and the QRPA ((R)QRPA) with their variances obtained with Brueckner short-range correlations. The effective weak coupling constant is assumed to be within the range $1.0 \leq g_{A}^{\text {eff }} \leq 1.25$ 32]. Here, $g_{A}^{\text {eff }}$ is the quenched axial-vector coupling constant. We note that the significant reduction (quenching) of the strength observed in nuclear Gamow-Teller transitions still has no clear experimental quantification and theoretical understanding. Usually, two possible physical origins of the quenching have been discussed in the literature: one due to the $\Delta$-isobar admixture in the nuclear wavefunction and another one due to the shift of the Gamow-Teller strength to higher excitation energies induced by short-range tensor correlations. In the absence of a better prescription, the effect of quenching is often simply evaluated by replacing the bare value $g_{A}=1.25$ with an empirical, quenched value $g_{A}^{\text {eff }}=1$.

Considering the ISM NMEs, the allowed intervals for half-lives are purely determined by the Majorana phase difference $\alpha_{12}$ with $0 \leq \sin ^{2} \alpha_{12} \leq 1$. From Fig. 2 we see that the allowed ranges of $T_{1 / 2}^{0 \nu}$ calculated with the QRPA, IBM and EDF NMEs differ significantly from the ISM ranges. For nuclei, where a comparison is possible, the ranges are slightly larger, however, shifted down to smaller half-lives. It reflects the difference in calculated NMEs between the ISM and a group of the QRPA, IBM and EDF approaches for $g_{A}=1.25$. We notice that for many nuclei the ranges of half-lives obtained with the (R)QRPA results by assuming a possible quenching of $g_{A}$ in nuclear matter (the third set of NMEs) are comparable with those of the QRPA, IBM and EDF NMEs for $g_{A}=1.25$. Thus, the problem of the correct description of two-nucleon short-range correlations and of the determination of the effective weak-coupling constant $g_{A}^{\text {eff }}$ is as important as the differences incorporated in the construction of nuclear wave functions. However, it is not clear yet how to determine $g_{A}^{e f f}$ reliably for a given nucleus [51].

In the last few years there has been a significant progress in understanding the source of the spread of calculated NMEs. Nevertheless, there is no consensus as yet among nuclear theorists on the correct NME values and their uncertainties. However, the recent development in the field is encouraging. There is good reason to believe that the uncertainty will be reduced.

We will finish with the following remarks:

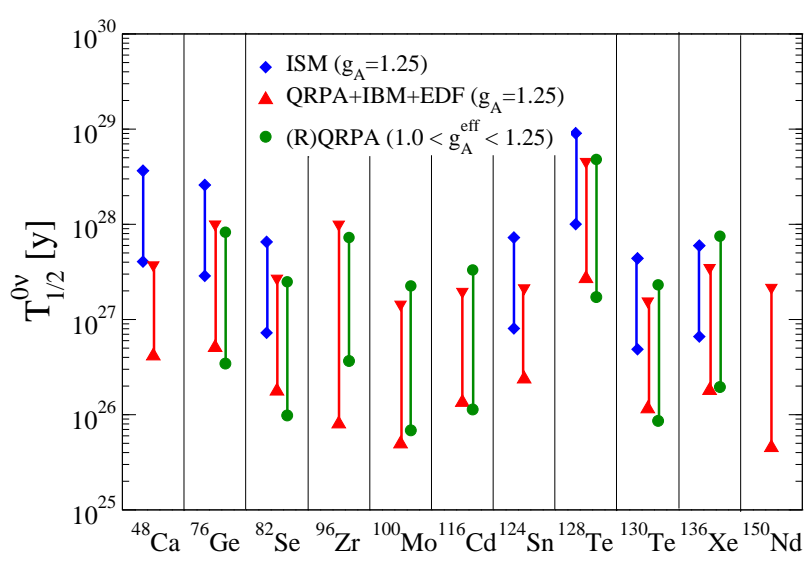

FIG. 2. (Color online) The allowed intervals of the $0 \nu \beta \beta$ decay half-life $T_{1 / 2}^{0 \nu}$ [see Eq. (22)] for nuclei of experimental interest in the case of the see-saw mechanism and inverted hierarchy of neutrino masses. Results are presented for the ISM and a group of 3 methods (QRPA, IBM and EDF) by considering $g_{A}=1.25$ and Jastrow short-range correlations. In addition the allowed (R)QRPA ranges of NMEs are considered by assuming $1.0 \leq g_{A}^{\text {eff }} \leq 1.25$ and the Brueckner short-range correlations [32].

1. We have considered the $0 \nu \beta \beta$-decay in the case of the inverted spectrum with $m_{0} \lesssim 5 \cdot 10^{-2} \mathrm{eV}$. If $m_{0} \gtrsim 1 \cdot 10^{-1} \mathrm{eV}$ the neutrino mass spectrum is quasi-degenerate

$$
m_{1} \simeq m_{2} \simeq m_{3}
$$

The effective Majorana mass is relatively large in this case and for both types of the neutrino mass spectrum is given by the expression

$$
m_{0}\left(\cos ^{2} \theta_{13} \cos 2 \theta_{12}-\sin ^{2} \theta_{13}\right) \leq\left|m_{\beta \beta}\right| \leq m_{0} .
$$

The allowed region for $m_{\beta \beta}$ is presented by the region between two parallel lines in the upper part of Fig1. It is evident that the Majorana mass mechanism of the $0 \nu \beta \beta$-decay can be checked also in the case of the quasi-degenerate spectrum if $m_{0}$ is known (from cosmological observations or from future $\beta$-decay experiments KATRIN [52, 53] and MARE[54]).

2. In the case of normal neutrino mass spectrum with $m_{0} \ll \sqrt{\Delta m_{S}^{2}} \simeq 8.7 \cdot 10^{-3} \mathrm{eV}$ we have normal mass hierarchy

$$
m_{1} \ll m_{2} \ll m_{3} .
$$

\footnotetext{
${ }^{7}$ For the sum of neutrino masses we have $\Sigma m_{i} \simeq 6 \cdot 10^{-2} \mathrm{eV}$ in this case.
} 
The effective Majorana mass is given in this case by the expression

$$
\begin{aligned}
& \left|m_{\beta \beta}\right| \simeq \\
& \left|\cos ^{2} \theta_{13} \sin ^{2} \theta_{12} \sqrt{\Delta m_{S}^{2}}+\sin ^{2} \theta_{13} \sqrt{\Delta m_{A}^{2}} e^{2 i \alpha_{23}}\right| \\
& \leq 3.9 \cdot 10^{-3} \mathrm{eV} .
\end{aligned}
$$

Thus, in the case of the normal mass hierarchy $\left|m_{\beta \beta}\right|$ is too small in order to be probed in the $0 \nu \beta \beta$-decay experiments of the next generation.

3. The check of the Majorana neutrino mass mechanism which we have discussed is possible even if an observation of the $0 \nu \beta \beta$-decay is made for only one isotope. If the half-lives of the $0 \nu \beta \beta$-decay of several isotopes will be measured and all measured half-lives satisfy the inequality (22), this will be additional evidence in favor of the Majorana mass mechanism. Let us also notice that if the NME problem will be finally solved than (independently of the character of the neutrino mass spectrum and the value of the lightest neutrino mass) an information about the Majorana neutrino mass mechanism could be obtained by comparing the values of the effective Majorana mass determined from the measured half-lives of different nuclei. If it would occur that the value of $\left|m_{\beta \beta}\right|$ does not depend on the nucleus it would be an argument in favor of the seesaw origin of Majorana neutrino masses.

\section{CONCLUSION}

From the standard seesaw mechanism, based on the assumption that the total lepton number is violated at the $\left(10^{14}-10^{15}\right) \mathrm{GeV}$ scale, follows that the Majorana neutrino exchange mechanism is the only mechanism of the neutrinoless double $\beta$-decay. In this case the half-life of the $0 \nu \beta \beta$-decay is given by the general expression (9). Thus, the test of the expression (9) could give us information about the validity of the standard seesaw mechanism. We have demonstrated that this check could be performed if $0 \nu \beta \beta$-decay will be observed in future experiments sensitive to the effective Majorana mass in the inverted mass hierarchy region, if a present cosmological bound on the sum of the neutrino mass will be improved down to $0.2 \mathrm{eV}$. For this case we calculated the allowed ranges of the half-lives of the $0 \nu \beta \beta$-decay of ${ }^{48} \mathrm{Ca}$, ${ }^{76} \mathrm{Ge}$ and other nuclei of experimental interest with the NMEs obtained in the framework of all methods existing at present. Of course, further progress in the calculation of the NMEs is desired to obtain more precise ranges for (22).

\section{ACKNOWLEDGMENTS}

The work of S.B. and W.P. is supported by the Deutsche Forschungsgemeinschaft (Transregio 27: Neutrinos and Beyond), the Munich Cluster of Excellence (Origin and Structure of the Universe), and the MaierLeibnitz-Laboratorium (Garching). A.F. and F.S. acknowledge support of the Deutsche Forschungsgemeinschaft within the project 436 SLK $17 / 298$ and of the VEGA Grant agency of the Slovak Republic under the contract No. 1/0639/09.
[1] R. Wendell et al. (Super-Kamiokande Collaboration), Phys. Rev. D 81, 09200 (2010).

[2] B. T. Cleveland et al. (Homestake), Astrophys. J. 496, 505 (1998); J. N. Abdurashitov et al. (SAGE Collaboration), Phys. Rev. C 80, 015807 (2009); W. Hampel et al. (GALLEX Colaboration), Phys. Lett. B 447, 127 (1999); M. Altmann et al. (GNO Collaboration), Phys. Lett. B 616, 174 (2005); P. Cravens et al. (Super-Kamiokande Collaboration), Phys. Rev. D 78, 032002 (2008); B. Aharmim et al. (SNO Collaboration), Phys.Rev.C81, 055504 (2010).

[3] A. Gando et al. (The KamLAND Collaboration), Phys. Rev. D 83, 052002 (2011).

[4] M.H.Ahn et al. (K2K Collaboration), Phys. Rev. D74, 072003 (2006).

[5] A.Habig et al. (MINOS Collaboration), Mod. Phys. Lett. A25, 1219 (2010).

[6] S.M. Bilenky, J. Hošek, and S.T. Petcov, Phys. Lett. B 94, 495 (1980).
[7] M. Gell-Mann, P. Ramond, and R. Slansky, in Supergravity, p. 315, edited by F. van Nieuwenhuizen and D. Freedman, North Holland, Amsterdam, 1979; T. Yanagida, Proc. of the Workshop on Unified Theory and the Baryon Number of the Universe, KEK, Japan, 1979 ; R.N. Mohapatra and G. Senjanović, Phys. Rev. Lett. 44, 912 (1980); P. Minkovski, Phys. Lett. B 67, 421 (1977).

[8] S. Weinberg, Phys. Rev. Lett. 43, 1571 (1979).

[9] B. Pontecorvo, J. Exptl. Theoret. Phys. 33, 549 (1957) [Sov. Phys. JETP 6, 429 (1958)]; J. Exptl. Theoret. Phys. 34, 247 (1958) [Sov. Phys. JETP 7, 172 (1958)].

[10] Z. Maki, M. Nakagawa, and S. Sakata, Prog. Theor. Phys. 28, 870 (1962).

[11] S. Davidson, E. Nardi, Y. Nir, Phys. Rept. 466, 105 (2008).

[12] E. Ma, Phys. Rev. Lett. 81, 1171 (1998).

[13] M. Doi, T. Kotani, and E. Takasugi, Progr. Theor. Phys. Suppl. 83, 1 (1985) .

[14] F. Simkovic, G. Pantis, J.D. Vergados, and A. Faessler, Phys. Rev. C 60, 055502 (1999). 
[15] V.A. Rodin, A. Faessler, F. Šimkovic, and P. Vogel, Phys. Rev. C 68, 044302 (2003); Nucl. Phys. A 793, 213 (2007), and erratum A 793, 213 (2007).

[16] F. Šimkovic, A. Faessler, V.A. Rodin, P. Vogel, and J. Engel, Phys. Rev. C 77, 045503 (2008).

[17] Dong-Liang Fang, A. Faessler, V. Rodin, and F. Šimkovic, Phys. Rev. C 82, 051301 (2010).

[18] J. Menéndez, A. Poves, E. Caurier, and F. Nowacki, Nucl. Phys. A 818, 139 (2009).

[19] M. Kortelainen, O. Civitarese, J. Suhonen, and J. Toivanen, Phys. Lett. B 647128 (2007); M. Kortelainen and J. Suhonen, Phys. Rev. C 75051303 (2007); Phys. Rev. C 76024315 (2007).

[20] P.K. Rath, R. Chandra, K. Chaturvedi, P.K. Raina, and J.G. Hirsch, Phys. Rev. C 82, 064310 (2010).

[21] J. Barea and F. Iachello, Phys. Rev. C 79, 044301 (2009).

[22] T.R. Rodriguez and G. Martinez-Pinedo, Phys. Rev. Lett. 105, 252503 (2010).

[23] A. Escuderos, A. Faessler, V. Rodin, and F. Šimkovic, J. Phys. G 37, 125108 (2010).

[24] G. A. Miller and J. E. Spencer, Ann. Phys. (NY) 100, 562 (1976).

[25] F. Šimkovic, A. Faessler, and P. Vogel, Phys. Rev. C 77, 015502 (2009).

[26] A.S. Barabash, Phys. Atom. Nucl. 70, 1191 (2007); F.T. Avignone, S.R. Elliott, and J. Engel, Rev. Mod. Phys. 80, 481 (2008); S.M. Bilenky, Phys. Part. Nucl. 41, 690 (2010).

[27] H. V. Klapdor-Kleingrothaus and I. V. Krivosheina, Mod. Phys. Lett. A 21, 1547 (2006)

[28] J. Jochum (GERDA Collaboration), Prog. Part. Nucl. Phys. 64, 261 (2010); S. Schönert (GERDA Collaboration), J. Phys. Conf. Ser. 203, 012014 (2010).

[29] L. Baudis et al. (Heidelberg-Moscow Collaboration), Phys. Rev. Lett. 83, 41 (1999).

[30] E. Andreotti et al. (CUORE Collaboration), Astropart. Phys. 34, 822 (2011); M.Sisti (CUORE Collaboration), J. Phys. Conf. Ser. 203, 012069 (2010).

[31] R. Arnold et al. (NEMO Collaboration), Phys. Rev. Lett. 95, 182302 (2005).

[32] F. Šimkovic, A. Faessler, H. Müther, V. Rodin, and M. Stauf, Phys. Rev. C 79, 055501 (2009).

[33] R. Gornea (EXO Collaboration), J. Phys. Conf. Ser. 259, 012039 (2010).
[34] V.M. Gehman (Majorana Collaboration), J. Phys. Conf. Ser. 136, 042047 (2008).

[35] R. Arnold et al. (SuperNEMO Colaboration), Eur. Phys. J. C 70, 927 (2010); L. Simard, Prog. Part. Nucl. Phys. 64, 270 (2010).

[36] Ch. Kraus et al., Prog. Part. Nucl. Phys. 64, 273 (2010); K. Zuber, AIP Conf. Proc. 942, 101 (2007).

[37] T. Schwetz, M. Tórtola, and J.W.F. Valle, New Journal of Physics 10, 113011 (2008).

[38] K. Abe et al. (T2K Collaboration), arXiv:1106.2822 [hep$\mathrm{ex}$.

[39] S.A. Thomas, F.B. Abdalla, and O. Lahav, Phys. Rev. Lett. 105, 031301 (2010)

[40] P.D. Serpico, Phys. Rev. Lett. 98, 171301 (2007).

[41] K.N. Abazajian et al., arXiv:1103.5083 [astro-ph].

[42] A. Dueck, W. Rodejohann, and K. Zuber, Phys. Rev. D 83, 113010 (2011).

[43] S. Hannestad, Prog.Part.Nucl.Phys. 65, 185 (2010).

[44] V. Tello et al., Phys. Rev. Lett. 106, 151801 (2011).

[45] R. Mohapatra and G. Senjanović, Phys. Rev. D 23, 165 (1981).

[46] F. Šimkovic, J. Vergados, and A. Faessler, Phys. Rev. D 82, 113015 (2010).

[47] A. Faessler, G.L. Fogli, E. Lisi, A.M. Rotunno, and F. Šimkovic, Phys. Rev. D 83, 113015 (2011).

[48] A. Faessler, A. Meroni, S.T. Petcov, F. Šimkovic, and J. Vergados, Phys. Rev. D 83, 113003 (2011).

[49] A. Ibarra, E. Molinaro, and S.T. Petcov, Phys. Rev. D 84, 013005 (2011).

[50] G. Senjanovic, arXiv:1012.4104 [hep-ph].

[51] A. Faessler, G.L. Fogli, E. Lisi, V. Rodin, and F. Šimkovic, Phys. Rev. D 79, 053001 (2009).

[52] A. Osipowicz et al. (KATRIN Collaboration), hep-ex/0109033 J. Angrik et al. (KATRIN Collaboration), KATRIN Design Report 2004, http://bibliothek.fzk.de/zb/berichte/FZKA7090.pdf

[53] E.W. Otten and C. Weinheimer, Rep. Prog. Phys. 71, 086201 (2008).

[54] E. Andreotti et al. MARE Collaboration, Nucl. Instrum. Meth. A 572, 208 (2007); A. Nucciotti et al. (MARE Collaboration), arXiv:1012.2290 [hep-ex]. 